\title{
Investigating the Wavelength Dependency of Dot Gain in Color Print
}

\author{
Mahziar Namedanian ${ }^{*} a$, Sasan Gooran ${ }^{a}$ and Daniel Nyström ${ }^{a}$ \\ ${ }^{a}$ Department of Science and Technology, Linköping University, \\ Norrköping Campus, Sweden
}

\begin{abstract}
By separating the optical dot gain from the physical dot gain, it is possible to study different behaviors of color inks on different papers. In this study we are investigating the dependency of dot gain and wavelength in color print. Microscopic images have been used to separate optical and physical dot gain from each other. The optical behavior of primary color inks in different absorbing wavelength bands has been studied. It has been illustrated that the light scattering in the paper is wavelength independent, and therefore the Point Spread Function which indicates the probability of light scattering of the paper does not change in visible wavelengths (380 $\mathrm{nm}-700$ $n m$ ). We have shown that it is possible to separate two printed color inks on one specific wavelength, due to the filtering behavior of the color inks. By considering the fact that light scattering in the paper is wavelength independent, it was possible to separately analyze the dot gain of each color.
\end{abstract}

Keywords: Color Ink, Dot Gain, Light Scattering, PSF

\section{INTRODUCTION}

Characterizing the halftone print properties, is useful for system calibration and quality control of the color reproduction. There are numerous physical phenomena which influence color perception such as the light source, surface reflection, light absorption, light scattering, multiple internal reflection at the ink-air interface, and the combination of several light absorbing and light scattering substances. ${ }^{1}$ Photon migrations within the paper from noninked to inked regions tend to increase the photon absorption and thus decrease the halftone reflectance. In this case the dots appear effectively larger than their physical size (also called Yule-Nielsen effect) which makes an accurate color prediction very difficult. ${ }^{2-4}$

Physical dot gain is caused by ink spreading around halftone dots. Several factors can contribute to the increase in halftone dot area. Different paper types have different ink absorption rates; uncoated papers can absorb more ink than coated ones. Printing pressure can squeeze the ink out of its dot shape causing gain, and ink viscosity is a contributing factor with coated papers; higher viscosity inks can resist the pressure better. ${ }^{5,6}$

The term light scattering refers to all physical processes that move apart photons in different directions. This phenomen is caused by variation of refractive index within a non-homogeneous medium. In 1871, Lord Rayleigh established the Rayleigh-scattering theory which is applied to independent scattering of particles that are about ten times smaller than wavelength of the incident light. ${ }^{1}$ In the Rayleigh theory the scattering coeficient depends on the wavelength of light, which means that the blue light is scattered more strongly than the red light. The scattered sunlight in the atmosphere is mostly blue, which explains why the sky is blue. In 1908, Gustav Mie proposed the theory that is generalized from the Rayleigh model. The Mie-scattering theory, ${ }^{7}$ which is derived from Maxwells' equations, can be compared by Rayleigh scattering theory by emphasizing on this fact that the light scattering is wavelength independent for mediums with particles bigger than the light wavelength. According to the Mie theory, the light scattering in the paper which is made by particles bigger than the light wavelengths is wavelength independent. Previously it has been shown that the photons with short wavelength can not penetrate in to deeper points of paper. Due to its strong power of scattering, the Modulation Transfer Function (MTF) of paper is higher for shorter wavelengths, however the difference is not signicant. ${ }^{8}$

The colors of halftone prints were predicted using different theories: the Murray-Davies model, the Neugebauer model, the Yule-Nielsen model, and Clapper-Yuel model for optical dot gain. In our previous study, ${ }^{9}$ we found

\footnotetext{
*E-mail: mahna@itn.liu.se, phone:(+46)11 363199; fax $(+46) 11363270$
} 
two models which are based on Murray-Davies model to separate the optical dot gain from the physical, by using both a spectrophotometer and a high resolution camera, which resulted in similar dot gain curves. Our high resolution camera is equipped with seven broadband filters, which gave this possibility to measure the dot gain in different wavelengths. In this study we have used the high resoluton approach to find the optical dot gain of color inks.

Since we have the possibility to separate the physical and optical dot gains, we can study the dependency of optical dot gain for different wavelengths. In this paper, by studying the optical dot gain for black ink (absorbing wavelength band of $400 \mathrm{~nm}-700 \mathrm{~nm}$ ) in different wavelengths, we show that light scattering of the paper is wavelength independent. However, since each color ink has its own absorbing wavelength band, which is different for different inks, the behavior of their dot gain can vary dependent on wavelength. The probability of a photon that enters the surface of the paper at a point and exits from another point of the paper is defined by Point Spread Function (PSF). ${ }^{11}$ We show that the light scattering in the paper is wavelength independent, which means that the PSF is independent of the ink and it doesn't change in different wavelengths.

The paper is organized as follows: In Section 2 we present briefly our approach using microscopic images to separate optical and physical dot gain. Section 3 investigates the behavior of optical dot gain for different inks with regards to the wavelengths of the visible spectrum. In section 4 , we study how to separate different color inks in two color print. Section 5 concludes the paper.

\section{SEPARATING OPTICAL FROM PHYSICAL DOT GAIN}

When the light is perpendicularly illuminated from below the paper, in the transmitted image of paper there is no optical dot gain effect. ${ }^{13,14}$ We have used this fact to approximate the physical dot gain. The model used in the current study are derived from Murray-Davies equation (Murray, 1936) [Eq. (1)].

$$
R_{m}=a_{e f f, R} R_{i}+\left(1-a_{e f f, R}\right) R_{p}
$$

Where $\left(R_{m}\right)$ is the measured reflectance value, $\left(a_{e f f, R}\right)$ is the effective dot area after print, $\left(R_{i}\right)$ is the reflectance value of the ink at full coverage, and $\left(R_{p}\right)$, is the reflectance value of the paper. The total dot gain $\left(\Delta a_{t o t}\right)$, is given by the difference between the effective $\left(a_{e f f}\right)$, and reference dot area, $\left(a_{r e f}\right)$.

$$
\Delta a_{t o t}=a_{e f f, R}-a_{r e f}
$$

The method which is used in this study to separate the physical dot gain from the total dot gain is to use transmitted images. The transmittance value is obtained from the light that is perpendicularly illuminated from underneath the paper. In this case the optical dot gain has no effect in the transmitted image. With the same logic, the physical dot gain $\left(\Delta a_{p h y}\right)$, will be calculated by the difference between the dot area $\left(a_{e f f, T}\right)$, and the reference one, $\left(a_{r e f}\right)$ [see Eq. (3)]. By subtracting the physical dot gain from the total dot gain, the optical dot gain is obtained [Eq. (4)].

$$
\begin{gathered}
\Delta a_{p h y}=a_{e f f, T}-a_{r e f} \\
\Delta a_{o p t}=\Delta a_{t o t}-\Delta a_{p h y}=a_{e f f, R}-a_{e f f, T}
\end{gathered}
$$

In this study, 21 patches with different coverage of cyan, magenta, yellow, and black have been printed. All the patches are halftoned by AM (150 lpi, $1200 \mathrm{dpi}$ ) and printed by commercial offset press (Heidelberg) on coated paper $\left(150 \mathrm{gr} / \mathrm{m}^{2}\right)$. The reference dot area coverage of the patches are $(0,5,10, \ldots, 95,100 \%)$.

Fig. 1(a) and Fig. 1(b) show the halftone patch at 35\% of black ink which is captured by the camera when the light was illuminated from above (reflected image) and below (transmitted image), respectively. In order to magnify the dots in these two figures, we are only showing a small part of the original image comparing to the mentioned image field of view. Because of the change of the dot coverage from one patch to another, the exposure time of high resolution camera should be changed. Therefore the camera is calibrated for each patch individually and the pixel value of paper and $100 \%$ ink is thereby changed from one patch to the next. We decided to place two narrow stripes of unprinted paper and $100 \%$ ink beside each patch to make sure that we use correct pixel values for paper and 100\% ink for each patch [see Fig.1]. The transmitted and reflected images 

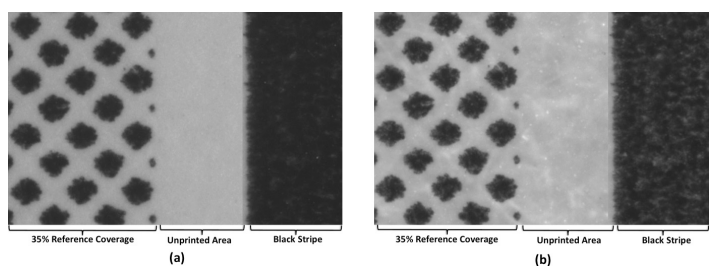

Figure 1. Micro scale images with 35\% reference coverage, (a) captured from above. (b) captured from below.

can be used to find the physical and the total dot gain, respectively. The reflectance value of the paper and the full tone coverage are computed from the average pixel values of the unprinted stripe and \%100 ink stripe, respectively. By replacing $R_{i}$ and $R_{p}$ with these average values in Eq.(1) and Eq.(2) the total dot gain $\left(\Delta a_{t o t}\right)$ is computed. By using transmitted images the paper value $T_{p}$ and the ink value $T_{i}$ are computed from the average pixel values of the unprinted stripe and \%100 ink stripe. With the same logic as above the physical dot gain will be calculated by the difference between the dot area $\left(a_{e f f, T}\right)$, and the reference one, $\left(a_{r e f}\right)$. Fig. 2 shows the total and physical dot gain of black ink, which are estimated using reflected and transmitted images, respectively.

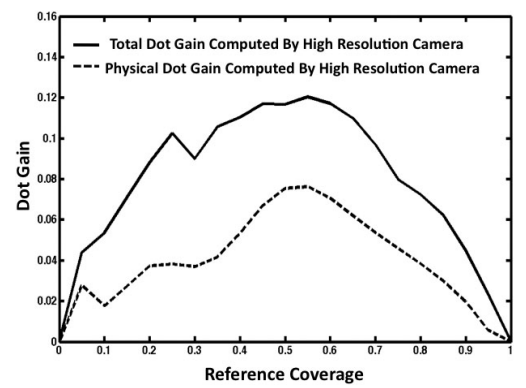

Figure 2. Total and physical dot gain of the black ink printed on a coated paper.

\section{ANALYSIS OF DOT GAIN IN DIFFERENT WAVELENGTHS}

The color inks, which are printed on a substrate, partially reflect the light in some wavelengths and absorb the rest. Therefore we can say that the color inks act as filter in the visible spectrum $(380 \mathrm{~nm}-700 \mathrm{~nm})$. In the human brain, the light wavelengths which are filtered by the ink and reflected from the paper are perceived as color. For instance, a cyan ink appears cyan, because it absorbs all the wavelengths of the white light, except the wavelengths we call cyan $(380 \mathrm{~nm}-550 \mathrm{~nm})$. Fig. 3 shows the absorbing and reflective wavelength band of cyan, magenta, and black. It is illustrated that the black ink absorb all the visible wavelengths but cyan and magenta only absorb some part of the visible wavelength.

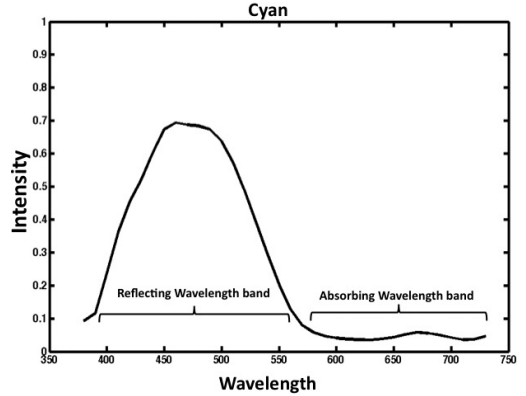

(a)

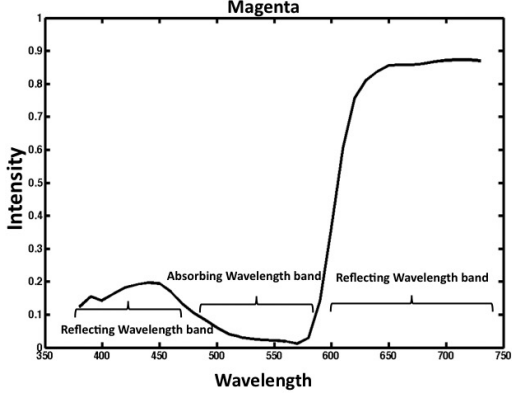

(b)

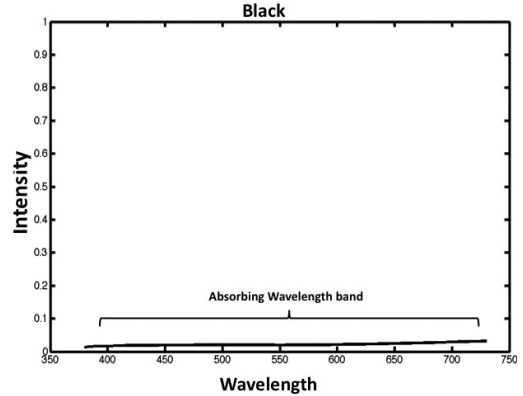

(c)

Figure 3. Absorbing and reflective wavelength band of color inks. (a)Cyan, (b)Magenta, (c)Black 


\subsection{Dot Gain of Color Inks in Different Wavelengths}

In this section we illustrate the dot gain behavior of color inks in different wavelengths. For this purpose, we used a high resolution camera $(1.9 \mu \mathrm{m} / \mathrm{pixel})$ with a field of view of $(2.7 \times 2 \mathrm{~mm})$, which is equipped with a set of seven broadband interference filters with center wavelengths band of $400 \mathrm{~nm}, 450 \mathrm{~nm}, 500 \mathrm{~nm}, 550 \mathrm{~nm}, 600 \mathrm{~nm}$, $650 \mathrm{~nm}$, and $700 \mathrm{~nm}$, in addition to the light source (a tungsten halogen lamp) (Fig. 4). All the interference filters have a bandwidth of $80 \mathrm{~nm}$, full width-half maximum (FWHM), except for the filter with $400 \mathrm{~nm}$, having $50 \mathrm{~nm}$ FWHM. The filters are located on the color filter wheel. By rotating the filter-wheel it is possible to illuminate the light in a specific wavelength band. ${ }^{15}$

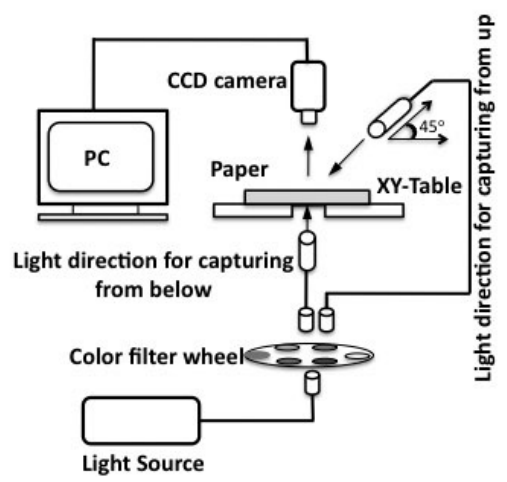

Figure 4. The high resolution camera setup for reflectance and transmittance imaging.

Fig. 5 shows the light intensity which is transmitted from interference-filters. The intensity of transmitted light, as it was promised by the manufacturer are between $55 \%-65 \%$ of the light source. The light, which is passed through the filters and is illuminated on the substrate, can be obtained by Eq. (5):

$$
I_{F_{n}}(\lambda)=I_{s}(\lambda) \cdot F_{n}
$$

$I_{F_{n}}(\lambda)$ represents the transmitted light through the filter and $I_{s}(\lambda)$ indicates the light source. The $(\lambda)$ variable indicates that $I_{F_{n}}(\lambda)$, and $I_{s}(\lambda)$ are a function of wavelength. $F_{n}$ indicates the characteristic of the filter where the subscript $(n)$ represents the filter number.

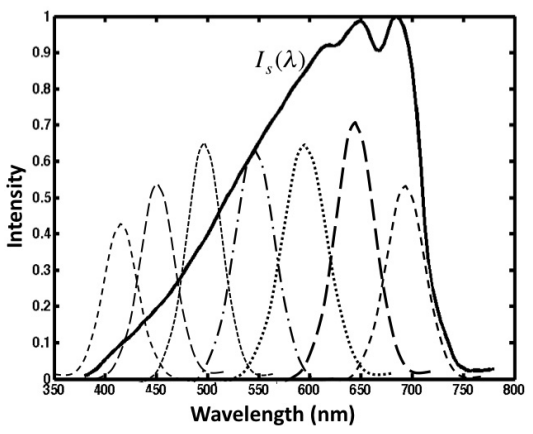

Figure 5. Transmittances of seven interference-filters

The light which is passed through the filter, gets illuminated on the ink layer. In the first step the color inks absorb some part of the incident light and transmit the rest. In the second step, the light which is transmitted through the ink comes to the paper layer and is scattered in the paper according to PSF function and reflected. The reflected light, is transmitted through the ink layer again. ${ }^{16,17}$ These steps can be expressed by the following equation:

$$
R_{F_{n}}(\lambda)=I_{F, n}(\lambda) \cdot\{t(\lambda) * P S F\} \cdot t(\lambda) \cdot R_{P}
$$

Where $(*)$ denotes the convolution operator and $(\cdot)$ gives element wise multiplication. The ink transmittance is denoted by $t(\lambda)$ and $R_{P}$ indicates the paper reflectance. $R_{F_{n}}(\lambda)$ is the reflectance value, when the $I_{F_{n}}(\lambda)$ 
is homogeneously illuminated on the paper and captured by high resolution camera. By putting $R_{F_{n}}(\lambda)$ in Murray-Davies equation [see Eq. (7), and Eq. (2)] it is also possible to calculate the total dot gain for each color inks in different wavelengths.

$$
a_{e f f}=\frac{R_{F_{n}}(\lambda)-R_{p, F_{n}}(\lambda)}{R_{i, F_{n}}(\lambda)-R_{p, F_{n}}(\lambda)}
$$

$R_{p, F_{n}}(\lambda)$, and $R_{i, F_{n}}(\lambda)$ represent the average of image-pixel values of unprinted stripe and $100 \%$ ink stripe. As mentioned before, we have printed 21 patches of each color ink (CMYK) on a coated paper. The yellow dot-pixel values which are printed on the paper are close to the paper-pixel values compare to the cyan and magenta. According to Eq. (7) the numerator and denominator of the fraction are both approaching zero, therefore it is hard to compute the dot gain of yellow ink in the wavelength between $(480 \mathrm{~nm}-700 \mathrm{~nm})$. Therefore in this paper we only consider the cyan, magenta, and black color inks to investigate the wavelength dependency of their dot gains.

Fig. 6(a), Fig. 6(b), and Fig. 6(c) show the total dot gain of cyan, magenta, and black in different absorbing wavelengths which are obtained by micro-scale image approach. It is illustrated that the total dot gains are different for cyan and magenta. However for black ink the total dot gains are quite close to each other, by a maximum difference less than $0.1 \%$ which is not significant. The total dot gain in Fig.6(c) is computed by using six interference filters. The black ink absorb all wavelengths in visible spectrum. The light source used in this experience had a low intensity in the wavelength band of $350 \mathrm{~nm}-400 \mathrm{~nm}$, therefore the first filter, with center wavelength of $400 \mathrm{~nm}$ is neglected.

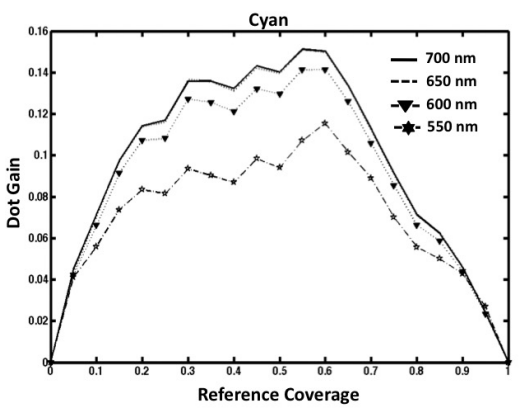

(a)

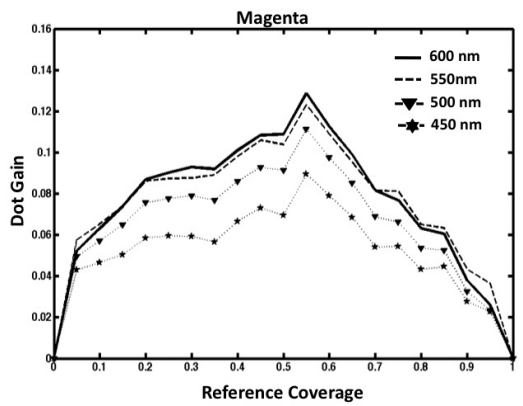

(b)

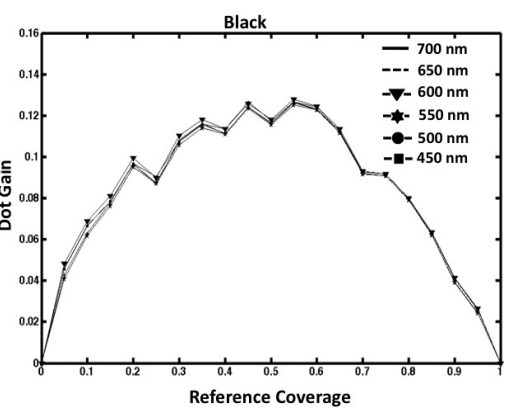

(c)

Figure 6. Total dot gains in the absorbing wavelength band. (a) Cyan ink (b) Magenta ink (c) Black ink

\subsection{Wavelength Dependency of Light Scattering}

The physical dot gain is a result of a real physical extension of ink dot. The wavelength of the light has no effect on the physical dot gain. Since in this study we are able to separate the physical and optical dot gain, we can study the dependency of optical dot gain for different wavelength bands.

Fig. 7 illustrates the total and optical dot gains for different wavelengths of black ink. It has been observed that the optical dot gain for different absorbing wavelength band are quite close to each other by a difference around $0.1 \%$. It can be observed that the difference between the optical dot gain of the area with small reference coverages are slightly larger than the area with bigger coverages. The reason is that the effective dot area $\left(a_{e f f}\right)$ for tiny dots are small and it is difficult to separate the physical dot gain from the total one, more accurately. Therefore it can be concluded that the optical dot gain curves are similar for all absorbing wavelength bands. In other words the optical dot gain is wavelength independent.

The light scattering of the paper, which is one of the factors creating optical dot gain, is also wavelength independent. Point Spread Function (PSF) is the probability of a photon that enters the surface of the paper at a point and exits from another point of the paper. ${ }^{11}$ The PSF is closely related to the optical properties of the paper. The wavelength independency of optical dot gain also concludes that PSF is wavelength independent.

Now the question is, why the total dot gain differs for color inks in different wavelength band. Use of the MurrayDavies model requires making the fundamental assumption that both the substrate and the ink are of uniform color. It is important to note that this is rarely the case. For typical printing on plain paper, dot densities 


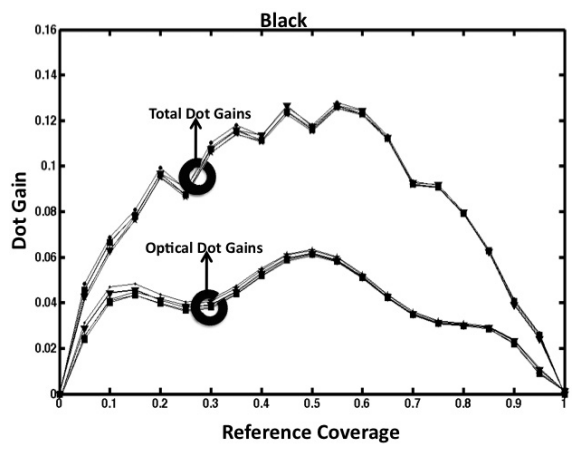

Figure 7. Total dot gain and optical dot gains for black ink in different wavelengths.

are not uniform. ${ }^{12}$ For a print without ink penetration, the reflected light passes twice through the thickness of ink layer. As the ink itself scatters the light, there is a significant loss in the light, therefore the behavior of ink transmittance in different wavelengths plays an important role in the reflectance value. To study the ink behavior in different wavelengths Eq.(8) is used to express the logarithmic form of the transmittance:

$$
D(\lambda)=-\log _{10}^{t(\lambda)}
$$

where $D(\lambda)$ is the density spectrum or absorption, which corresponds to the transmittance $t(\lambda)$ and it can be measured by densitometer. In the density scale, $D(\lambda) \approx 0$ corresponds to the reflective wavelength band of the ink. The values of $D(\lambda)$ increase logarithmically in the absorbing wavelength band. An extreme case for the ideal ink is when the absorbing wavelength band corresponds to an infinite density, $D(\lambda) \approx \infty$. Fig.8(a) and Fig.8(b) show a density spectrum of cyan and magenta. The ink density is changing in the absorbing wavelength band. Therefore according to Eq.(8) we can say that the ink transmittance is not constant in the absorbing wavelength band.

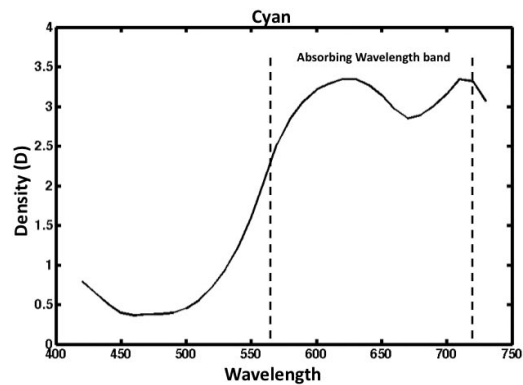

(a)

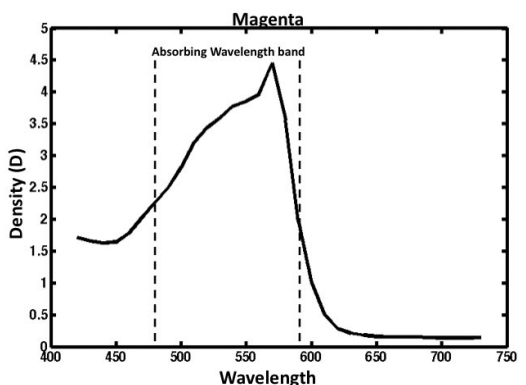

(b)

Figure 8. Ink density in different wavelengths. (a) Cyan (b) Magenta

According to Eq.(6) the reflected light $\left(R_{F_{n}}(\lambda)\right)$ from the printed ink on the paper depends on two factors; PSF of paper and ink transmittance. As mentioned before the light scattering in the paper is wavelength independent. The total dot gain $\left(\Delta a_{t o t}\right)$, which is derived from $R_{F_{n}}(\lambda)$, differs for different wavelengths, due to the variation of ink transmittance values in the absorbing wavelength band. In other words, the color inks are not quite ideal in the absorbing wavelength band. The total dot gain for the ideal color inks should be equal for all absorbing wavelengths like black ink.

\section{HOW TO SEPARATE TWO-PRINTED COLORS}

In the reflective wavelength band of a color, the ink doesn't absorb any part of the light and the color-ink dots will invisible from the paper. This method can be used to separate different color inks in two color print. The secondary colors (RGB) are produced when the primary colors (CMY) are printed two by two. Fig.3 shows the reflective wavelength band of the primary colors. By illuminating the light in the reflective wavelength band 
of one of the two colors which are printed on each other, it is possible to neglect that color and have only the second one.

Fig.9 shows the yellow and cyan dots which are printed by AM halftoning method on a coated paper. By illuminating the light in $700 \mathrm{~nm}$ (the reflective wavelength band of yellow), the yellow dots will vanish and only the cyan dots will remain. By illuminating the light in $400 \mathrm{~nm}$, which is the cyan reflective wavelength band, we can similarly remove the cyan dots. Fig.9(c) shows the dot gain curve of the green patch and the cyan patch which are obtained by illuminating the light in $700 \mathrm{~nm}$. It is observable that the cyan dot gain which remained from green patch is close to the dot gain of only cyan patch, by the difference less than $0.1 \%$. Here for better illustration, the dot-off-dot printing model have been used. In this study the dot gain is computed for two color inks which are printed on top of each other (dot-on-dot). In this case when two color inks are printed on coated paper the results are the same. However for uncoated paper, penetration is a factor which might change the result.

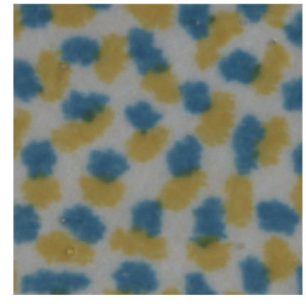

(a)

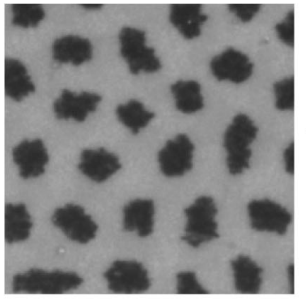

(b)

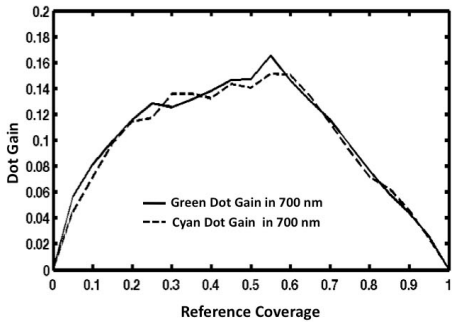

(c)

Figure 9. (a): Cyan and yellow dots printed by dot-off-dot halftoning method on a coated paper. (b): The same patch that captured in $700 \mathrm{~nm}$. (c) Dot gain of green (cyan and yellow) and only cyan ink in $700 \mathrm{~nm}$

Since each color ink has its own absorbing wavelength band, the behavior of its dot gain can vary dependent on the wavelength. For all color inks we have calculated dot gain in two steps: 1 . Without any filter 2 . With the seven interference filters. Fig.10 shows that among all filters, the filter corresponding to maximum dot gain in the absorbing wavelength band of specific ink has almost the same dot gain value as when we are not using any filter. For cyan, magenta, and black the maximum difference between the dot gain at absorbing wavelength band and the dot gain using no filter are only $0.4 \%, 0.6 \%$, and $0.1 \%$, respectively. Here we show that the dot gain of the color ink under the daily light is equivalent to the dot gain in the absorbing wavelength band. Therefore by illuminating the incident light in the defined wavelengths on the images, which are produced by the two inks, it is possible to characterize the ink behavior (optical dot gain, physical shape of the dots, and etc) in each image individually.

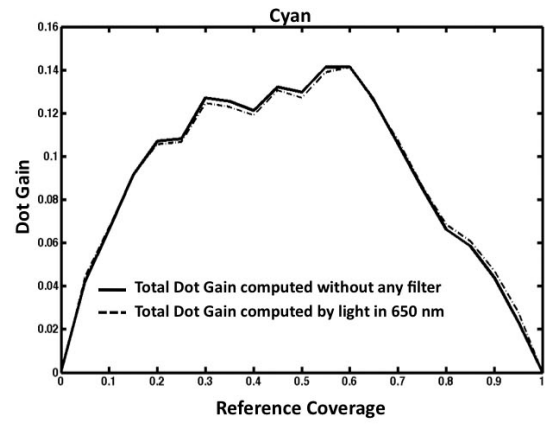

(a)

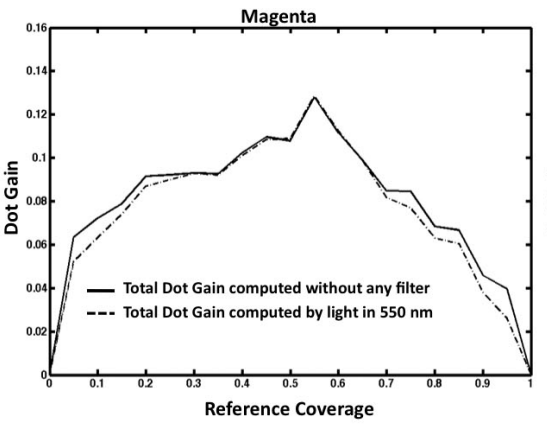

(b)

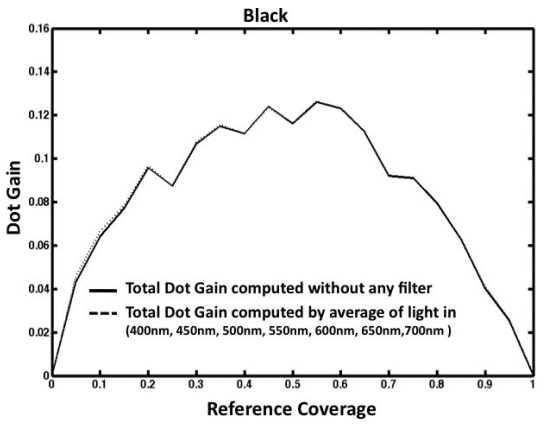

(c)

Figure 10. Dot gain is calculated in two steps: 1-without any filter, 2-with filter. (a)Cyan, (b)Magenta,(c) Black 


\section{CONCLUSION}

The method which is used in this paper to separate the physical and optical dot gain, is based on the captured images which are taken by a high resolution camera. We used this method to investigate the behavior of dot gain in different wavelength band for different color inks. The results show the behavior of optical dot gain for different wavelengths, which indicates that the light scattering in the paper is wavelength independent. Due to the variation of ink transmittance values in the absorbing wavelength band, the total dot gain differs for different wavelengths. We can also say that the PSF for different wavelengths does not change. By separating the two primary colors which are printed on a paper it is possible to determine the behavior of each color individually. This can be used to predict color reproduction more accurately.

\section{REFERENCES}

[1] G. Sharma, "Handbook Digital Color Imaging," Webster, New York, 2003. http://www.crcnetbase.com/doi/book/10.1201/9781420041484.

[2] R. D. Hersch, P. Emmel, F. Collaud, and F. Crete, "Spectral reflection and dot surface prediction models for color halftone prints," in J. Electronic. Imaging, vol. 14, No. 3, 3300, 2005.

[3] R. Geoffrey, "Optical dot gain in a halftone print : Tone reproduction and image quality in the graphic arts," in Journal of imaging science and technology, ISSN 1062-3701, 1997.

[4] L. Yang, R.Lenz, and B.Kruse, "Light scattering and ink penetration effects on tone reproduction," in J. Opt. Soc. Am. A, Vol. 18, No.2, 360-366, 2001.

[5] M. Sormaz, T. Stamm, S. Mourad, and P.Jenny, "Stochastic modeling of light scattering with fluorescence using a Monte Carlo-based multiscale approach," J. Opt. Soc. Am. A 26, No. 6, 1403-1413, (2009).

[6] J. S. Arney, C. D. Arney, M. Katsube, and P. G. Engeldrum, "An MTF analysis of paper," J. Imaging Sci. Technol, 40, No. 1, 19-25, (1996).

[7] G. Mie, "Beitrge zur Optik trber medien, speziell kolloidaler Metallsungen," Annalen der Physik, vierte Folge, Band 25, 377445, 1908 (in German).

[8] M. Ukishima, H. Kaneko, T. Nakaguchi, N, Tsumura, M. Hauta-Kasri, J. Parkkinen, and Y. miyake,, "A Simple Method to Measure MTF of Paper and Its Application for Dot Gain Analysis," IEICE TRANSACTIONS on Fundamentals of Electronics, Communications and Computer Sciences,E92, No. 12, 3328-3335, 2009.

[9] M. Namedanian, and S. Gooran, "High Resolution Analysis of Optical and Physical Dot Gain," in TAGA conference proceeding, 48-51, 2010.

[10] M. Ukishima, Y. Suzuki, N. Tsumura, T. Nakaguchi, M. Mkinen and J. Parkkinen, "A Method to Separately Model Mechanical and Optical Dot Gain Effects in Color Halftone Prints ," in TAGA conference proceeding, 16-18, 2010.

[11] S. Gustavson, "Dot Gain in Color Halftones," Ph.D Disertation No.492, Dept. of Electrical Engineering, Linkoping University, Sweden, 1997.

[12] D. R. Wyble, and R. S. Berns , "A Critical Review of Spectral Models Applied to Binary Color Printing," COLOR research and application, 25, No. 1, 4-19, 2000.

[13] C. Koopipat, N. Tsumura, M. Fujino, and Y. Miyake, "Effect of Ink Spread and Opitcal Dot Gain on the MTF of Ink Jet Image," J. Imaging Sci. Technol, 46, No. 4, 321-325, 2002.

[14] 1. C. Koopipat, 2. N. Tsumura, 3. M. Fujino, and 4. Y. Miyake, "Prediction of Image Reflectance Based on the Measurement of Mechanical and Optical Dot Gain," J. Japan Hardcopy, 2002, 584-585, 2002.

[15] D. Nyström, "High Resolution Analysis of Halftone Prints," Linköping Studies in Science and Technology Dissertations, No.1229, 2008.

[16] S. Inoue, "Analyzing CTF of Print by MTF of Paper" J. Imaging Sci. Technol, 42, No. 6, 572-576, 1998.

[17] P. G. Engeldrum, "Paper Substrate Spread Function and the MTF of Photographic Paper" J. Imaging Sci. Technol, 48, No. 1, 50-57, 2004. 